\title{
Does age affect the test performance of secondary sonographic findings for pediatric appendicitis?
}

\author{
Jeffrey T. Neal ${ }^{1}$ (I) $\cdot$ Michael C. Monuteaux ${ }^{1} \cdot$ Shawn J. Rangel ${ }^{2} \cdot$ Richard G. Bachur $^{1} \cdot$ Carol E. Barnewolt $^{3}$
}

Received: 22 October 2020 / Revised: 29 March 2021 / Accepted: 2 May 2021 / Published online: 27 May 2021

(C) The Author(s), under exclusive licence to Springer-Verlag GmbH Germany, part of Springer Nature 2021

\begin{abstract}
Background Secondary sonographic findings of appendicitis can aid image analysis and support diagnosis with and without visualization of an appendix.

Objective We sought to determine if age affected the test performance of secondary findings for pediatric appendicitis.

Materials and methods We performed a medical record review of emergency department patients younger than 19 years of age who had a sonogram for suspected appendicitis. Our primary patient outcome was appendicitis, as determined by pathology or by image-confirmed perforation/abscess. Our primary analysis was test performance of secondary sonographic findings as recorded by sonographers on the final diagnosis of appendicitis stratified by age ( $<6$ years, 6 to $<11$ years, 11 to $<19$ years).

Results A total of 1,219 patients with suspected appendicitis were evaluated by ultrasound, and 1,147 patients met the criteria for analysis. Of the 1,147 patients, $431(37.6 \%)$ had a final diagnosis of appendicitis. Across all age groups, echogenic fat was the most accurate secondary finding (92.5\% [95\% confidence interval (CI): 91.0, 94.0]) and free fluid was the least accurate secondary finding $(54.7 \%$ [95\% CI: 51.8, 57.5]). There was no significant difference in the age-stratified test performance of secondary sonographic findings except that (1) appendicolith was a more accurate predictor in patients $<6$ years old than in the middle group $(P<0.001)$ or the oldest group $(P<0.001)$, and $(2)$ free fluid was a more accurate predictor in the middle group than in the oldest group $(P=0.02)$.

Conclusion There are no significant differences in the age-stratified test performance of secondary sonographic findings in the prediction of pediatric appendicitis except that appendicolith is more predictive in younger patients.
\end{abstract}

Keywords Age $\cdot$ Appendicitis $\cdot$ Appendix $\cdot$ Children $\cdot$ Secondary findings $\cdot$ Ultrasound

\section{Introduction}

Sonography has become an essential component of the diagnostic evaluation for pediatric appendicitis because it is noninvasive and does not expose the patient to ionizing radiation. When the entire appendix is visualized, primary factors intrinsic to the appendix itself such as compressibility, appendiceal wall

Jeffrey T. Neal

jeffrey.neal@childrens.harvard.edu

1 Division of Emergency Medicine, Boston Children's Hospital, BCH 3066, 300 Longwood Ave., Boston, MA 02115, USA

2 Department of Surgery, Boston Children's Hospital, Boston, MA, USA

3 Department of Radiology, Boston Children's Hospital, Boston, MA, USA thickness and appendiceal diameter are incorporated into the diagnostic interpretation of appendicitis. However, the frequency of incomplete and/or non-visualization of the appendix varies widely depending on operator experience and patient factors, and is sometimes as high as $60 \%$ [1-4]. Clinical scoring systems and laboratory data, such as white blood cell (WBC) count or absolute neutrophil count (ANC), can also be integrated into decision making, but these confer limited diagnostic yield [5]. Occasionally, the sonographic diagnosis of appendicitis remains equivocal despite visualization of an appendix. Secondary sonographic findings such as free fluid, echogenic fat, regional hyperemia or an obstructing or extruded appendicolith may assist in the diagnosis [6-9]. Similarly, the absence of these secondary sonographic findings may be sufficient to exclude the diagnosis of appendicitis [10-12]. Structured ultrasound (US) evaluation for secondary findings, in combination with sonographer training aimed at searching for these findings, has been shown to provide more meaningful US interpretations [13], and 
standardized reporting has been shown to decrease reliance on computed tomography (CT) as well as admissions for observation $[14,15]$. When the appendix is visualized on US, but appendicitis cannot be confirmed, this may lead to additional cross-sectional imaging, transfer to centers with available pediatric surgeons, or hospitalization for clinical monitoring. Because US is the primary imaging modality in the evaluation of pediatric appendicitis, it is important to optimize its application through the awareness and detection of secondary signs.

Many studies have evaluated the addition of clinical judgment, laboratory values or combinations of secondary US findings in the diagnostic evaluation for appendicitis. To our knowledge, there are no pediatric studies that have evaluated the effect of age on the test performance of secondary US findings of appendicitis. Younger children tend to have less reliable signs and symptoms and present at later stages as evidenced by a higher frequency of perforation [16]. Therefore, more advanced disease may allow for greater reliance on secondary sonographic characteristics with or without visualization of an appendix.

Our goal was to determine the age-related test characteristics and prevalence of secondary sonographic findings in the diagnosis of pediatric appendicitis. We hypothesized that secondary sonographic findings may be more diagnostically important in evaluating younger children with suspected appendicitis. We sought to evaluate the test characteristics of secondary sonographic findings overall as well as in cases when the appendix was not visualized or was incompletely seen.

\section{Materials and methods}

\section{Study design and setting}

We performed a retrospective review of medical records of pediatric patients younger than 19 years of age who presented to a single urban academic pediatric emergency department between November 2015 and October 2018 and who had US ordered to evaluate suspected appendicitis. In general, patients are evaluated by utilizing the institutional clinical pathway for appendicitis, which involves a screening of complete blood cell count and calculation of a Pediatric Appendicitis Score (PAS). For patients with a PAS greater than or equal to four, the pathway suggests a sonogram as the initial diagnostic modality, although this is ultimately left up to clinician discretion [17]. The emergency department has approximately 60,000 patient visits annually. The institutional review board of our center approved this study.

\section{Study population}

Participants were identified by a previously developed radiology database implemented within the department of radiology in the beginning of November 2015, with the goal of standardizing the terminology used to describe sonographer observations when performing sonograms for suspected appendicitis. All patients who had US ordered for evaluation of appendicitis between Nov. 1, 2015, and Oct. 31, 2018, in the emergency department were included in the study. The questionnaire was completed by the sonographer performing the US at the time of the exam. All sonograms were performed under the supervision of an attending radiologist, though the attending did not necessarily personally scan the patient. The questionnaire was available to, but was not systematically reviewed or edited by, the faculty radiologist after sonography completion. A majority of sonograms (about 70\%) were scanned by five exclusively pediatric sonographers, highly trained by pediatric radiologists with a special interest in sonography. This group of sonographers had an experience range of between 3 and 15 years, with a median of 8.8 years.

Patients 19 years of age and older and patients with a history of relevant abdominal surgery (such as appendectomy or ventriculoperitoneal shunt placement) were excluded from study participation. In addition, four patients were excluded as the final outcome of appendicitis could not be confirmed.

\section{Methods}

Data were abstracted electronically from the radiology data tool and uploaded to a Research Electronic Data Capture (REDCap) database. The following data elements were abstracted: demographics (age and sex), date of visit, sonography data (exam date, sonographer name, quality of sonographic window) and primary US findings including visualization of the appendix, the appendiceal tip, maximal appendiceal diameter and compressibility. Visualization of the appendix was defined as visualization of part or all of the appendix or a candidate appendix (a blind-ending tubular structure in the expected anatomical region) as deemed by the interpreting radiologist (Figs. 1 and 2). Templated descriptors mutually agreed upon between the Department of Radiology, the Department of Surgery and the Division of Emergency Medicine before the creation of the radiology data tool were used to standardize the description of positive, negative and equivocal studies.

Recorded secondary findings of appendicitis included free fluid, regional hyperemia, appendicolith and echogenic fat (Figs. 3, 4, 5 and 6). Free fluid was defined as any volume of fluid in the pelvis, echo-free or echogenic, outside of the bowel lumen, either adjacent to or remote from the right lower quadrant/traditional appendix location. Hyperemia was defined as the subjective appearance of hypervascularity as compared to adjacent muscle (typically the psoas muscle or anterior abdominal wall musculature) when a color/power Doppler window is applied and gain/sensitivity settings are optimized. Appendicolith was defined as an echogenic structure, casting a posterior acoustic shadow, with a convex, nearfield margin, either within or outside the lumen of the 


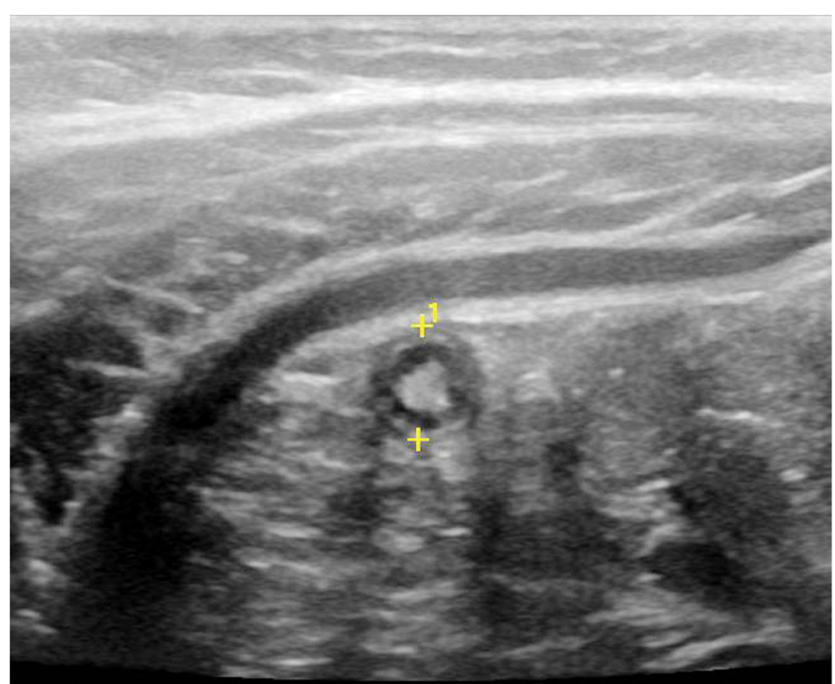

Fig. 1 Transverse ultrasound image shows a normal appendix (cursors) in a 5-year-old girl

candidate appendix. Echogenic fat was defined as a complete rind of homogeneously bright soft tissue, without a gap, surrounding the perimeter of the candidate appendix, or as subjectively increased echogenicity of the right lower quadrant as compared to left lower quadrant fat. For each element, the findings were recorded as present, absent or maybe. Free text comments could be added. For the purposes of this analysis, if the secondary finding was recorded as maybe or no comment, it was assumed to be present or absent, respectively. Additional variables such as ethnicity, race, weight, height (when available), duration of symptoms (hours), WBC count, ANC, US imaging impression, additional cross-sectional imaging impressions (when obtained) and position of the appendix (retrocecal/not retrocecal) were manually extracted by record review.

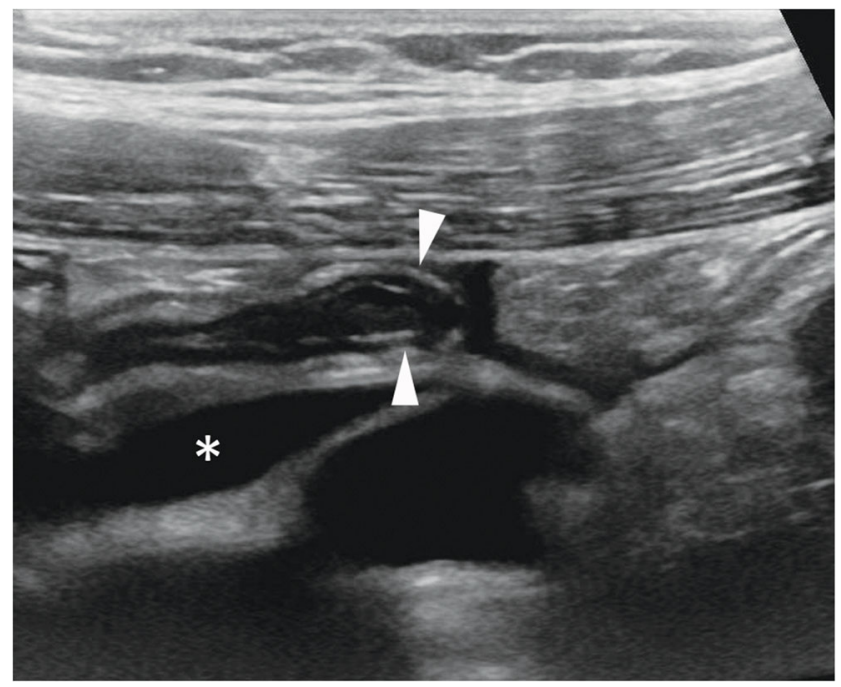

Fig. 2 Normal appendix in a 16-year-old boy. Longitudinal ultrasound image shows a blind-ending appendiceal tip (arrowheads) with free fluid (asterisk)

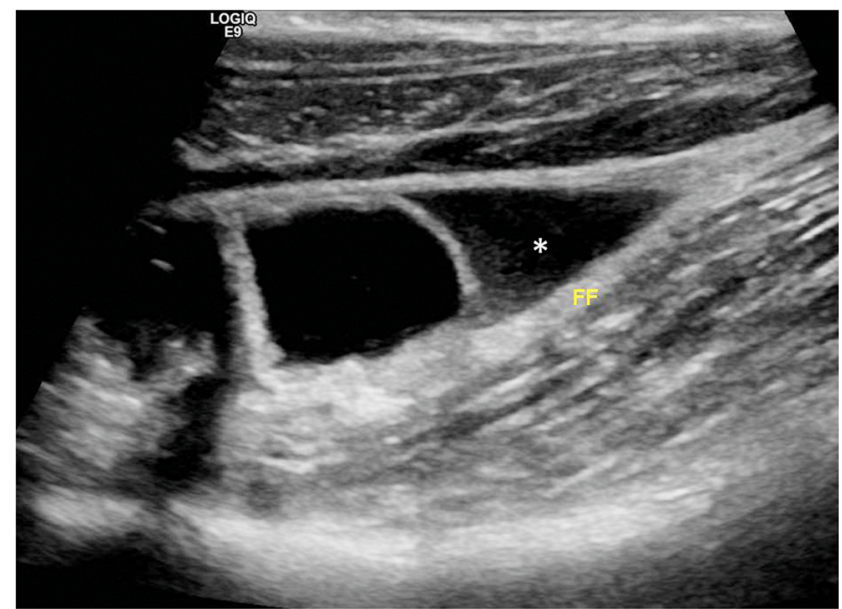

Fig. 3 Acute appendicitis in a 10-year-old boy. Longitudinal ultrasound image shows free fluid ( $F F$, asterisk) adjacent to a loop of fluid-filled bowel

Patient management variables such as operative versus nonoperative management were also obtained. If the patient underwent an operation, pathology was reviewed and the case was divided into the following categories: (1) no appendicitis, (2) mild/early/tip/mucosal infiltrate only, (3) moderate/full thickness inflammation or (4) severe/gangrene/perforation/necrosis. If the patient did not have an operation, the case was delineated as follows: (1) no appendicitis, discharged from the emergency department; (2) no appendicitis, if hospitalized for serial exams and discharged in improved condition or with an alternative diagnosis; or (3) imaged-confirmed appendicitis, hospitalized, antibiotics only, discharged. If the patient was discharged from the emergency department, the institution's electronic medical record was reviewed for any follow-up visits within 14 days of the initial US. Although every patient

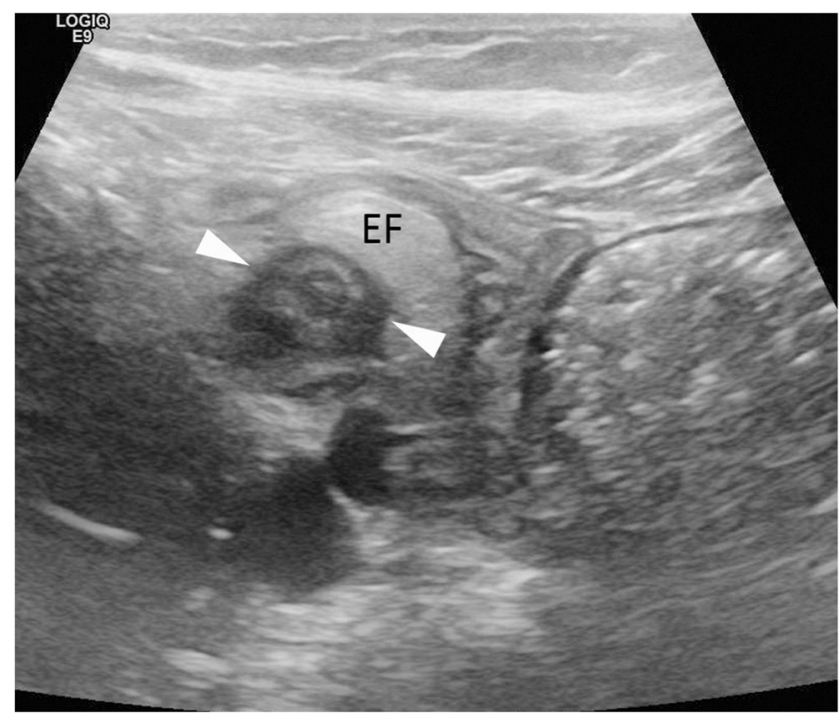

Fig. 4 Acute appendicitis in an 18-year-old woman. Transverse ultrasound image shows the appendix (arrowheads) surrounded by prominent echogenic fat $(E F)$ 


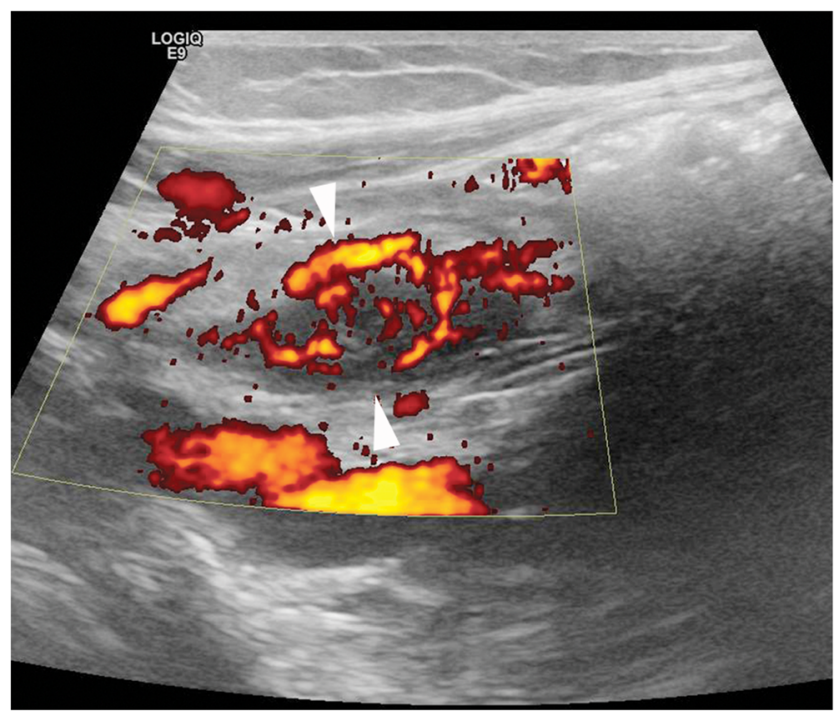

Fig. 5 Acute appendicitis in an 18-year-old woman. Longitudinal power Doppler ultrasound image shows the appendix (arrowheads) with hyperemia of the appendiceal wall and the surrounding echogenic fat

received a follow-up chart review, if no additional outpatient visits or emergency department visits were noted in the chart, this was recorded as unconfirmed follow-up.

\section{Outcomes}

Our primary outcome was a diagnosis of appendicitis (either based on surgical pathology or perforation or abscess based on US or additional cross-sectional imaging). Perforation was classified by either (1) a well-defined fluid collection in the region of the appendix on US or additional cross-sectional imaging or (2) visualized perforation during the operation or on gross pathology. The diagnosis was further classified into

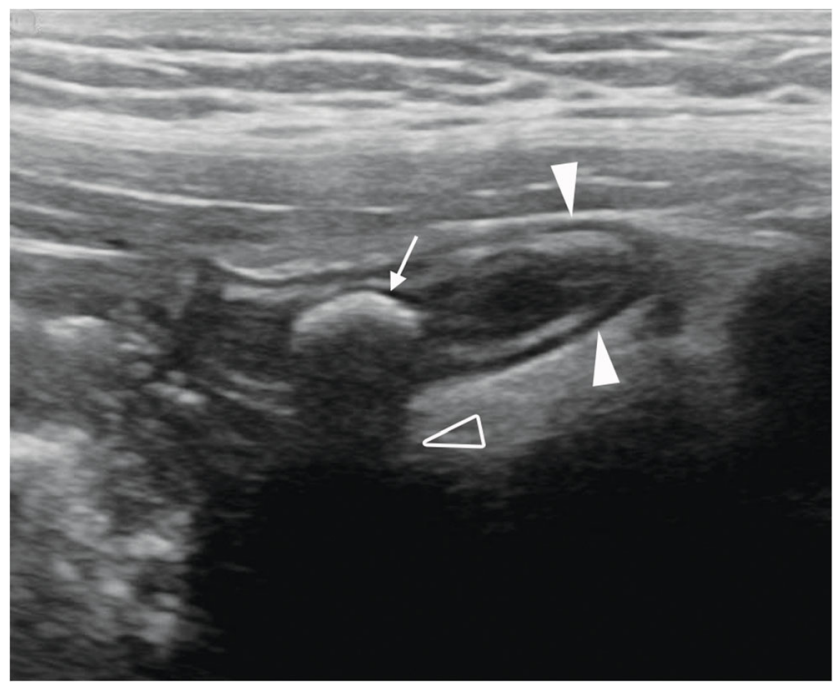

Fig. 6 Acute appendicitis in a 15-year-old girl. Longitudinal ultrasound image shows an inflamed appendiceal tip (solid arrowheads) with an appendicolith (arrow) casting a sharp acoustic shadow (open arrowhead) uncomplicated versus complicated appendicitis (abscess at the time of diagnosis or within 14 days, prolonged hospitalization $>2$ calendar days at the initial visit or readmission within 14 days, parenteral antibiotics $>2$ days in the hospital or at home). Our primary analysis of interest was the test performance of secondary sonographic findings to detect the final diagnosis of appendicitis, overall and stratified by age. The age groups ( $<6$ years, 6 to $<11$ years and 11 to $<19$ years) were determined a priori to attempt to primarily divide into common developmental categories (i.e. young child, school-age, adolescence), which have been previously described in the literature given age-dependent clinical presentations of appendicitis $[18,19]$, and secondarily to maximize statistical power with relatively similar sample sizes between groups.

\section{Data analysis}

Since this was a retrospective review of a previously collected data instrument, we aimed to include the entire population of patients during the study period to provide maximal precision. Descriptive statistics were used to characterize the demographic and clinical features of the study population. Test characteristics with $95 \%$ confidence intervals (CIs) were calculated for primary and secondary US findings, including sensitivity, specificity, negative predictive value, positive predictive value, overall accuracy and the area under the receiver operating characteristic curve, both in the total sample and within strata of age group. Risk difference calculations with $95 \%$ CIs were performed to determine the significance to compare test characteristics between age groups. A subgroup analysis was also conducted to evaluate the test characteristics of secondary US findings among the patients in whom the appendix was described as not visualized. There are many definitions of equivocal or nondiagnostic US in the literature $[6,20]$; however, non-visualization of the appendix was believed to allow for the analysis of the impact of secondary sonographic findings in the absence of any primary findings. All statistical analyses were performed utilizing STATA (version 14.1; StataCorp, College Station, TX) and SPSS (version 23; IBM, Armonk, NY).

\section{Results}

\section{Characteristics of study subjects}

During the study period, 1,219 patients underwent a sonogram for appendicitis that included tabulation of discrete findings by a pediatric sonographer on an electronic radiology questionnaire. Fifty-one patients were excluded due to age and 17 patients were excluded due to a history of relevant abdominal surgery. Four patients with presumed uncomplicated appendicitis did not have operative care or imaging evidence of perforation so they were excluded due the uncertainty 
surrounding an official diagnosis per the study definition of appendicitis, leaving a total of 1,147 patients in the study. Of these patients, only $50 / 1,147$ patients $(4.4 \%)$ had a no comment designation with regard to compressibility (when the appendix was visualized) or the presence or absence of an appendicolith. The majority of patients were ages 11 to younger than 19 years $(48.6 \%$ [558/1,147]). Of the 1,147 patients, $357(31.1 \%)$ were referred from an outside facility, though all, including the $59.4 \%(212 / 357)$ who had previous sonograms, had onsite sonograms once in our emergency department. The summary statistics of this population are presented in Table 1 .

\section{Main results}

Prevalence and test characteristics of primary sonographic findings are presented in Online Supplementary Material 1. The appendix was visualized completely or partially in $82.4 \%$ $(945 / 1,147)$ of cases. Twenty-five pediatric sonographers performed at least one US examination (mean: 46, range: 1,322) with five sonographers who tended to work evening and night shifts performing $68 \%(781 / 1,147)$ of the scans.

Of the 1,147 patients included in the study, $431(37.6 \%)$ had an ultimate diagnosis of appendicitis, of which 131 (30.4\%) were subclassified as complicated appendicitis (Table 2). Cases of complicated appendicitis were much more common in the youngest age group $(64.1 \%$ [25/39]) than in the middle age group (35.5\% [61/172]) or the oldest age group (20.5\% [45/
220]) ( $P<0.001$ and $P<0.001$, respectively). The overall frequency of negative appendectomy was $5.0 \%$ (20/400).

Secondary sonographic findings within the study population are presented in Table 3. Free fluid was most commonly seen in $63.4 \%(727 / 1,147)$ of cases and an appendicolith was least commonly seen in $18.0 \%(206 / 1,147)$ of cases. Echogenic fat had the highest test performance overall (sensitivity $=94.2 \%$, specificity $=91.5 \%$ ) and across all age groups. Free fluid had the lowest test performance overall (sensitivity $=74.0 \%$, specificity $=43.0 \%$ ) and across all age groups. Observation of an appendicolith was more predictive of appendicitis in the youngest age group ( $<6$ years) when compared to the middle age group $(6$ to $<11$ years, $P<0.001)$ and to the oldest age group $(11$ to $<19$ years; $P<0.001)$. Free fluid was more predictive of appendicitis in the middle age group versus the oldest age group $(P=0.023)$. Test performance of the secondary sonographic characteristics among those without perforation is available in Online Supplementary Material 2. Overall, in the study population, there were no statistically significant differences in the age-stratified test performance of the individual secondary findings for a diagnosis of pediatric appendicitis except in the scenarios described above.

Visualization rates of the appendix did not vary significantly between age groups. Within the subset of 202 cases where the appendix was described as not visualized on US (Table 4), there were only 16 cases of appendicitis. The prevalence of appendicitis within this subset $(7.9 \%)$ was lower than the
Table 1 Patient characteristics $(n=1,147)$

\begin{tabular}{ll}
\hline Characteristic & Value \\
\hline Age group & \\
$<6$ y & $172 / 1,147(15.0 \%)$ [mean: $4.6 \pm 1.0]$ \\
6 to $<11$ y & $417 / 1,147(36.4 \%)$ [mean: $8.6 \pm 1.4]$ \\
11 to $<19$ y & $558 / 1,147(48.6 \%)$ [mean: $14.4 \pm 2.1]$ \\
Gender (female) & $566 / 1,147(49.3 \%)$ \\
Ethnicity (not Hispanic or Latino) & $822 / 1,147(71.7 \%)$ \\
Race (Caucasian) & $660 / 1,147(57.5 \%)$ \\
Duration of symptoms & \\
$0-12$ h & \\
$>12-24$ h & $192 / 1,147(16.7 \%)$ \\
$>24-36$ h & $207 / 1,147(18.0 \%)$ \\
$>36-48$ h & $233 / 1,147(20.3 \%)$ \\
$>48-72$ h & $24 / 1,147(2.1 \%)$ \\
$>72-96$ h & $160 / 1,147(13.9 \%)$ \\
$>96-120$ h & $110 / 1,147(9.6 \%)$ \\
$>120$ h & $69 / 1,147(6.0 \%)$ \\
Transfer from outside hospital $(n=357,31.1 \%)$ & $152 / 1,147(13.3 \%)$ \\
Previous ultrasound & \\
Previous computed tomography & \\
Previous magnetic resonance imaging & $212 / 357(59.4 \%)$ \\
\hline
\end{tabular}

$h$ hours, $y$ years of age 
Table 2 Final patient outcome

\begin{tabular}{|c|c|c|c|c|}
\hline Characteristic & $\begin{array}{l}\text { Overall } \\
(n=1,147)\end{array}$ & $\begin{array}{l}<6 \mathrm{y} \\
(n=172)\end{array}$ & $\begin{array}{l}6 \text { to }<11 \text { y } \\
(n=417)\end{array}$ & $\begin{array}{l}11 \text { to }<19 y \\
(n=558)\end{array}$ \\
\hline Appendicitis & $37.6 \%(431 / 1,147)$ & $22.7 \%(39 / 172)$ & $41.2 \%(172 / 417)$ & $39.4 \%(220 / 558)$ \\
\hline Complicated & $30.4 \%(131 / 431)$ & $64.1 \%(25 / 39)$ & $35.5 \%(61 / 172)$ & $20.5 \%(45 / 220)$ \\
\hline Operative & $88.2 \%(380 / 431)$ & $76.9 \%(30 / 39)$ & $86.0 \%(148 / 172)$ & $91.8 \%(202 / 220)$ \\
\hline Nonoperative & $11.8 \%(51 / 431)$ & $23.1 \%(9 / 39)$ & $14.0 \%(24 / 172)$ & $8.2 \%(18 / 220)$ \\
\hline \multicolumn{5}{|l|}{ Operative pathology } \\
\hline Mild & $25.8 \%(103 / 400)$ & $8.6 \%(3 / 35)$ & $19.9 \%(31 / 156)$ & $33.0 \%(69 / 209)$ \\
\hline Moderate & $43.3 \%(173 / 400)$ & $25.7 \%(9 / 35)$ & $44.9 \%(70 / 156)$ & $45.0 \%(94 / 209)$ \\
\hline Severe & $26.0 \%(104 / 400)$ & $51.4 \%(18 / 35)$ & $30.1 \%(47 / 156)$ & $18.7 \%(39 / 209)$ \\
\hline Negative & $5.0 \%(20 / 400)$ & $14.3 \%(5 / 35)$ & $5.1 \%(8 / 156)$ & $3.3 \%(7 / 209)$ \\
\hline No appendicitis & $62.4 \%(716 / 1,147)$ & $77.3 \%(133 / 172)$ & $58.8 \%(245 / 417)$ & $60.6 \%(338 / 558)$ \\
\hline Hospitalized, serial exams & $20.5 \%(147 / 716)$ & $25.6 \%(34 / 133)$ & $21.6 \%(53 / 245)$ & $17.8 \%(60 / 558)$ \\
\hline No appendicitis, follow-up confirmed & $48.0 \%(344 / 716)$ & $36.1 \%(48 / 133)$ & $44.1 \%(108 / 245)$ & $55.6 \%(188 / 558)$ \\
\hline No appendicitis, unable to confirm follow-up & $28.6 \%(205 / 716)$ & $34.6 \%(46 / 133)$ & $31.0 \%(76 / 245)$ & $24.6 \%(83 / 558)$ \\
\hline
\end{tabular}

${ }^{\mathrm{a}}$ Includes negative appendectomy patients

$y$ years of age

Table 3 Age-stratified test characteristics of secondary sonographic findings in the prediction of appendicitis

\begin{tabular}{|c|c|c|c|c|}
\hline Characteristic & $\begin{array}{l}\text { Overall } \\
(n=1,147)\end{array}$ & $\begin{array}{l}<6 \mathrm{y} \\
(n=172)\end{array}$ & $\begin{array}{l}6 \text { to }<11 \text { y } \\
(n=417)\end{array}$ & $\begin{array}{l}11 \text { to }<19 \text { y } \\
(n=558)\end{array}$ \\
\hline \multicolumn{5}{|l|}{ Free fluid ${ }^{\mathrm{a}}$} \\
\hline Prevalence & $63.4 \%(727 / 1,147)$ & $59.9 \%(103 / 172)$ & $65.2 \%(272 / 417)$ & $63.1 \%(352 / 558)$ \\
\hline Sensitivity & $74.0 \%(69.6-78.1 \%)$ & $76.9 \%(60.7-88.9 \%)$ & $79.7 \%(72.9-85.4 \%)$ & $69.1 \%(62.5-75.1 \%)$ \\
\hline Specificity & $43.0 \%(39.4-46.7 \%)$ & $45.1 \%(36.5-54.0 \%)$ & $44.9 \%(38.6-51.4 \%)$ & $40.8 \%(35.5-46.3 \%)$ \\
\hline PPV & $43.9 \%(40.2-47.6 \%)$ & $29.1 \%(20.6-38.9 \%)$ & $50.4 \%(44.3-56.5 \%)$ & $43.2 \%(37.9-48.5 \%)$ \\
\hline NPV & $73.3 \%(68.8-77.5 \%)$ & $87.0 \%(76.7-93.9 \%)$ & $75.9 \%(68.1-82.6 \%)$ & $67.0 \%(60.1-73.4 \%)$ \\
\hline \multicolumn{5}{|l|}{ Appendicolith $^{\mathrm{a}}$} \\
\hline Prevalence & $18.0 \%(206 / 1,147)$ & $16.3 \%(28 / 172)$ & $18.9 \%(79 / 417)$ & $17.7 \%(99 / 558)$ \\
\hline Sensitivity & $40.6 \%(35.9-45.4 \%)$ & $53.9 \%(37.2-69.9 \%)$ & $39.0 \%(31.6-46.7 \%)$ & $39.6 \%(33.0-46.3 \%)$ \\
\hline Specificity & $95.7 \%(93.9-97.0 \%)$ & $94.7 \%(89.5-97.9 \%)$ & $95.1 \%(91.6-97.4 \%)$ & $96.5 \%(93.9-98.2 \%)$ \\
\hline PPV & $85.0 \%(79.3-89.5 \%)$ & $75.0 \%(55.1-89.3 \%)$ & $84.8 \%(75.0-91.9 \%)$ & $87.9 \%(79.8-93.6 \%)$ \\
\hline NPV & $72.8 \%(69.8-75.6 \%)$ & $87.5 \%(81.0-92.4 \%)$ & $68.9 \%(63.7-73.8 \%)$ & $71.0 \%(66.6-75.1 \%)$ \\
\hline \multicolumn{5}{|l|}{ Hyperemia } \\
\hline Prevalence & $43.5 \%(499 / 1,147)$ & $37.2 \%(64 / 172)$ & $47.5 \%(198 / 417)$ & $42.5 \%(237 / 558)$ \\
\hline Sensitivity & $91.7 \%(88.6-94.1 \%)$ & $97.4 \%(86.5-99.9 \%)$ & $95.5 \%)$ & $90.5 \%(85.8-94.0 \%)$ \\
\hline Specificity & $85.5 \%(82.7-88.0 \%)$ & $80.5 \%(72.7-86.8 \%)$ & $83.7 \%(78.4-88.1 \%)$ & $88.8 \%(84.9-91.9 \%)$ \\
\hline PPV & $79.2 \%(75.3-82.6 \%)$ & $59.4 \%(46.4-71.5 \%)$ & $79.8 \%(73.5-85.2 \%)$ & $84.0 \%(78.7-88.4 \%)$ \\
\hline NPV & $94.4 \%(92.4-96.1 \%)$ & $99.1 \%(95.0-100 \%)$ & $93.6 \%(89.5-96.5 \%)$ & $93.5 \%(90.2-95.9 \%)$ \\
\hline \multicolumn{5}{|l|}{ Echogenic fat } \\
\hline Prevalence & $40.7 \%(467 / 1,147)$ & $26.7 \%(46 / 172)$ & $45.3 \%(189 / 417)$ & $41.6 \%(232 / 558)$ \\
\hline Sensitivity & $94.2 \%(91.6-96.2 \%)$ & $94.9 \%(82.7-99.4 \%)$ & $95.4 \%(91.0-98.0 \%)$ & $93.2 \%(89.0-96.1 \%)$ \\
\hline Specificity & $91.5 \%(89.2-93.4 \%)$ & $93.2 \%(87.5-96.9 \%)$ & $89.8 \%(85.3-93.3 \%)$ & $92.0 \%(88.6-94.7 \%)$ \\
\hline PPV & $86.9 \%(83.5-89.9 \%)$ & $80.4 \%(66.1-90.6 \%)$ & $86.8 \%(81.1-91.3 \%)$ & $88.4 \%(83.5-92.2 \%)$ \\
\hline NPV & $96.3 \%(94.6-97.6 \%)$ & $98.4 \%(94.4-99.8 \%)$ & $96.5 \%(93.2-98.5 \%)$ & $95.4 \%(92.5-97.4 \%)$ \\
\hline
\end{tabular}

For sensitivity, specificity, positive predictive value (PPV) and negative predictive value (NPV), 95\% confidence intervals are given in parentheses. $y$ years of age ${ }^{a}$ Appendicolith more accurate as predictor for appendicitis in $<6$ y vs. 6 to $<11$ y $(P<0.001)$ and vs. 11 to $<19$ y $(P<0.001)$. Free fluid more accurate as a predictor for appendicitis in 6 to $<11$ y vs. 11 to $<19$ y $(P=0.023)$ 
Table 4 Test characteristics of secondary sonographic findings in the prediction of appendicitis when the appendix is described as not visualized

\begin{tabular}{|c|c|c|c|c|}
\hline Characteristic & $\begin{array}{l}\text { Overall } \\
(n=202)\end{array}$ & $\begin{array}{l}<6 \mathrm{y} \\
(n=31)\end{array}$ & $\begin{array}{l}6 \text { to }<11 \text { y } \\
(n=66)\end{array}$ & $\begin{array}{l}11 \text { to }<19 \text { y } \\
(n=105)\end{array}$ \\
\hline Appendicitis & $7.9 \%(16 / 202)$ & $0.0 \%(0 / 31)$ & $12.1 \%(8 / 66)$ & $7.6 \%(8 / 105)$ \\
\hline \multicolumn{5}{|l|}{ Free fluid } \\
\hline Prevalence & $58.9 \%(119 / 202)$ & $54.8 \%(17 / 31)$ & $62.1 \%(41 / 66)$ & $58.1 \%(61 / 105)$ \\
\hline Sensitivity & $93.8 \%(69.8-99.8 \%)$ & $-{ }^{\mathrm{a}}$ & $100 \%(63.1-100 \%)$ & $87.5 \%(47.4-99.7 \%)$ \\
\hline Specificity & $44.1 \%(36.8-51.5 \%)$ & $45.1 \%(27.3-64.0 \%)$ & $43.1 \%(30.2-56.8 \%)$ & $44.3 \%(34.2-54.8 \%)$ \\
\hline PPV & $12.6 \%(10.8-14.7 \%)$ & $0 \%(0-19.5 \%)$ & $19.5 \%(16.2-23.3 \%)$ & $11.5 \%(8.6-15.1 \%)$ \\
\hline NPV & $98.9 \%(92.4-99.8 \%)$ & $100 \%(76.8-100 \%)$ & $100 \%(87.7-100 \%)$ & $97.7 \%(87.2-99.6 \%)$ \\
\hline \multicolumn{5}{|l|}{ Appendicolith } \\
\hline Prevalence & $5.0 \%(10 / 202)$ & $0 \%(0 / 31)$ & $6.1 \%(4 / 66)$ & $5.7 \%(6 / 105)$ \\
\hline Sensitivity & $37.5 \%(15.2-64.6 \%)$ & $-{ }^{\mathrm{a}}$ & $25.0 \%(3.2-65.1 \%)$ & $50.0 \%(15.7-84.3 \%)$ \\
\hline Specificity & $97.9 \%(94.6-99.4 \%)$ & $100 \%(88.8-100 \%)$ & $96.6 \%(88.1-99.6 \%)$ & $97.9 \%(92.8-99.8 \%)$ \\
\hline PPV & $60.0 \%(32.0-82.7 \%)$ & & $50.0 \%(14.0-86.0 \%)$ & $66.7 \%(30.1-90.3 \%)$ \\
\hline NPV & $94.8 \%(92.6-96.4 \%)$ & $100 \%(88.8-100 \%)$ & $90.3 \%(86.2-93.3 \%)$ & $96.0 \%(92.2-97.9 \%)$ \\
\hline \multicolumn{5}{|l|}{ Hyperemia } \\
\hline Prevalence & $13.4 \%(27 / 202)$ & $12.9 \%(4 / 31)$ & $16.7 \%(11 / 66)$ & $11.4 \%(12 / 105)$ \\
\hline Sensitivity & $56.3 \%(29.9-80.3 \%)$ & $-{ }^{\mathrm{a}}$ & $62.5 \%(24.5-91.5 \%)$ & $50.0 \%(15.7-84.3 \%)$ \\
\hline Specificity & $90.3 \%(85.1-94.2 \%)$ & $87.1 \%(70.2-96.4 \%)$ & $89.7 \%(78.8-96.1 \%)$ & $91.8 \%(84.4-96.4 \%)$ \\
\hline PPV & $33.3 \%(21.3-48.1 \%)$ & & $45.5 \%(24.8-67.8 \%)$ & $33.3 \%(16.1-56.6 \%)$ \\
\hline NPV & $96.0 \%(93.2-97.7 \%)$ & $100 \%(87.2-100 \%)$ & $94.6 \%(87.6-97.7 \%)$ & $95.7 \%(91.7-97.8 \%)$ \\
\hline \multicolumn{5}{|l|}{ Echogenic fat } \\
\hline Prevalence & $12.9 \%(26 / 202)$ & $0 \%(0 / 31)$ & $16.7 \%(11 / 66)$ & $14.3 \%(15 / 105)$ \\
\hline Sensitivity & $68.8 \%(41.3-89.0 \%)$ & $-{ }^{\mathrm{a}}$ & $62.5 \%(24.5-91.5 \%)$ & $75.0 \%(34.9-96.8 \%)$ \\
\hline Specificity & $91.9 \%(87.1-95.4 \%)$ & $100 \%(88.8-100 \%)$ & $89.7 \%(78.8-96.1 \%)$ & $90.7 \%(87.1-95.7 \%)$ \\
\hline PPV & $42.3 \%(29.0-56.9 \%)$ & & $45.5 \%(24.8-67.8 \%)$ & $40.0 \%(24.1-58.3 \%)$ \\
\hline NPV & $97.2 \%(94.3-98.6 \%)$ & $100 \%(88.8-100 \%)$ & $94.6 \%(87.6-97.7 \%)$ & $97.8 \%(93.0-99.3 \%)$ \\
\hline
\end{tabular}

For sensitivity, specificity, positive predictive value (PPV) and negative predictive value (NPV), 95\% confidence intervals are given in parentheses. $y$ years of age

${ }^{\text {a }}$ Unable to be calculated due to zero cases of appendicitis in group

overall prevalence of appendicitis within the study group (37.6\%). Free fluid was the most sensitive $(93.8 \%$ [95\% CI: $69.8,99.8])$ and appendicolith was the most specific $(97.9 \%$ [95\% CI: 94.6, 99.4]) predictor for appendicitis in this group. Of the 78 cases with absence of secondary sonographic findings, none was diagnosed with appendicitis. Thirteen of the 16 cases of appendicitis had 2 or more secondary sonographic findings of appendicitis, and as the number of secondary findings increased, the likelihood of appendicitis increased (Online Supplementary Material 3).

\section{Discussion}

Our study investigated the age-stratified test performance of secondary sonographic findings in the prediction of pediatric appendicitis. The prevalence of secondary findings did not vary significantly between the different age groups. Echogenic right lower quadrant fat was the most predictive secondary sonographic finding for appendicitis across all age groups, which confirms similar findings in other studies of pediatric [21-23] and adult appendicitis [24]. The sonographic visualization of an appendicolith was more accurate for the diagnosis of appendicitis in the youngest age group as compared to the older two age groups. This may bear some relation to the higher frequency of complicated appendicitis (64.1\% [25/39]) in this age group, which has also been previously reported in the literature [16, 25-27].

The appendix was partially or completely visualized in $82.4 \%(945 / 1,147)$ of cases, with similar visualization frequencies across all age groups $(81.2 \%$ [453/558] to $84.2 \%$ [351/417]). Although non-visualization of the appendix is not the only criterion for nondiagnostic US, we evaluated this particular subset of patients to analyze the impact of secondary sonographic findings in the absence of primary findings such as appendiceal diameter or compressibility. Seventy-eight of the 202 non-visualized cases had no secondary sonographic findings and were not diagnosed with appendicitis, suggesting that, 
in the absence of secondary findings, one could potentially rule out appendicitis when the appendix is not visualized. Similarly, in a recent study by Wiersma et al. [10], of the 20\% (41/212) of study patients with non-visualization of the appendix and no evidence of secondary signs, none developed acute appendicitis. In the remaining non-visualized cases, appendicolith, echogenic fat or hyperemia were highly specific; therefore, if the pretest probability of appendicitis is high based on other historical, physical and laboratory findings, these findings may push one to pursue additional cross-sectional imaging. Nevertheless, the sample of non-visualized cases is small and the study is likely underpowered to make these conclusions.

Structured US evaluation for appendicitis and associated secondary sonographic findings as well as standardized reporting of these findings have previously led to improved visualization rates and less utilization of additional cross sectional imaging [13-15]. Our study had a visualization rate that was relatively high as compared to rates reported in the literature $[1,4]$. This study was performed at an academic institution with sonographers and radiologists familiar with pediatric patients and secondary sonographic findings, so further study should be directed at dissemination of structured US evaluation and recognition of these findings to referring facilities.

Our study has several limitations. First, although the data were prospectively collected during each US examination, this is a retrospective study. Therefore, we are limited to the data and the images collected on the initial US studies. Although a standardized radiology tool for describing US findings was used, we did not review the images from each US to ensure standardized application of these descriptors. This was a sonographer-generated database, and although the questionnaire was available in the electronic medical record, it was not systematically edited or reviewed. While about $70 \%$ of the sonograms were performed by 5 dedicated pediatric sonographers with a median of 8.8 years of experience at our facility, it is possible the radiologists' interpretation of secondary findings may have differed from the sonographers. Although this is acknowledged as a limitation, it increasingly reflects the workflow of busy imaging departments where direct radiologist scanning of all patients with concern for appendicitis is not routine.

Second, for purposes of analysis of secondary findings, we assumed that maybe equaled present and no comment equaled absent (which could indicate no evaluation was performed or the finding was not seen). This could potentially under- or overestimate the effect of each secondary finding; however, a designation of no comment never exceeded more than $2.1 \%$ $(24 / 1,147)$ of the study population for each secondary finding.

Third, the population is highly subject to selection bias. Out of the study population, $31.1 \%(357 / 1,147)$ were referred into the study site due to concern for appendicitis, and 59.4\% (212/ 357 ) of those referred patients had a previous abdominal US. Given that the pretest probability of appendicitis is higher in this subset of patients, the sonographers and radiologists may have been inclined to spend more time attempting to visualize the appendix and/or secondary findings. However, the sonographers were not necessarily aware of historical details, perhaps minimizing this potential bias.

Fourth, we were limited to our institutional chart review for follow-up purposes. Although every patient had a follow-up chart review, 28.6\% (205/716) of patients had no additional outpatient or emergency department visits within our institution and were assumed to not have appendicitis within 14 days. It is possible that a limited number of these patients returned to a different institution and were diagnosed with appendicitis.

Finally, this is a single-center study staffed with pediatric sonographers and pediatric radiologists available $24 \mathrm{~h}$ a day, which is not universal at many institutions. The visualization frequencies of the appendix $(82.4 \%[945 / 1,147]$ in our study population) and secondary findings are much higher than those reported at non-pediatric institutions and community hospitals. Nevertheless, in the literature, appendix visualization at some institutions is reported as high as $91.7 \%$ [28]. Furthermore, 5 of 25 sonographers (the evening and night shift team) performed $68 \%(781 / 1,147)$ of the right lower quadrant scans, further skewing the institution-specific bias. Therefore, the impact of these secondary findings on the ultimate diagnosis of pediatric appendicitis may not be easily extrapolated to other settings.

\section{Conclusion}

Secondary sonographic findings are valuable factors in the diagnosis of appendicitis in pediatric patients, and appear to be strong predictors even in the absence of direct visualization of the appendix. In the majority of secondary sonographic findings, there is no statistically significant difference in the age-stratified test performance for pediatric appendicitis. However, the observation of an appendicolith is a more accurate predictor of appendicitis in the youngest of patients.

Supplementary Information The online version contains supplementary material available at https://doi.org/10.1007/s00247-021-05100-0.

Acknowledgments Data collection for this study was partially funded by an internal grant, the Dr. Michael Shannon Emergency Medicine Award (Boston Children's Hospital). The abstract of this article was previously accepted as a poster presentation at the Proceedings of the Pediatric Academic Societies Annual Meeting for presentation in May 2020, which was canceled due to the coronavirus disease 2019 (COVID-19) pandemic. The authors would like to formally thank all of the dedicated, hardworking sonographers whose never-ending attention to detail and tireless compassion inspire us to improve our skills and strive for excellence.

\section{Declarations}

Conflicts of interest None 


\section{References}

1. Ross MJ, Liu H, Netherton SJ et al (2014) Outcomes of children with suspected appendicitis and incompletely visualized appendix on ultrasound. Acad Emerg Med 21:538-542

2. Mittal MK, Dayan PS, Macias CG et al (2013) Performance of ultrasound in the diagnosis of appendicitis in children in a multicenter cohort. Acad Emerg Med 20:697-702

3. Schuh S, Man C, Cheng A et al (2011) Predictors of non-diagnostic ultrasound scanning in children with suspected appendicitis. J Pediatr 158:112-118

4. Cohen B, Bowling J, Midulla P et al (2015) The non-diagnostic ultrasound in appendicitis: is a non-visualized appendix the same as a negative study? J Pediatr Surg 50:923-927

5. Benabbas R, Hanna M, Shah J, Sinert R (2017) Diagnostic accuracy of history, physical examination, laboratory tests, and point-ofcare ultrasound for pediatric acute appendicitis in the emergency department: a systematic review and meta-analysis. Acad Emerg Med 24:523-551

6. Partain KN, Patel A, Travers C et al (2016) Secondary signs may improve the diagnostic accuracy of equivocal ultrasounds for suspected appendicitis in children. J Pediatr Surg 51:1655-1660

7. Telesmanich ME, Orth RC, Zhang W et al (2016) Searching for certainty: findings predictive of appendicitis in equivocal ultrasound exams. Pediatr Radiol 46:1539-1545

8. Estey A, Poonai N, Lim R (2013) Appendix not seen: the predictive value of secondary inflammatory sonographic signs. Pediatr Emerg Care 29:435-439

9. Mirza WA, Naveed MZ, Khandwala K (2018) Utility and accuracy of primary and secondary ultrasonographic signs for diagnosing acute appendicitis in pediatric patients. Cureus 10:e3779

10. Wiersma F, Toorenvliet BR, Bloem JL et al (2009) US examination of the appendix in children with suspected appendicitis: the additional value of secondary signs. Eur Radiol 19:455-461

11. Nah SA, Ong SS, Lim WX et al (2017) Clinical relevance of the nonvisualized appendix on ultrasonography of the abdomen in children. J Pediatr 182:164-169.e1

12. Held JM, McEvoy CS, Auten JD et al (2018) The non-visualized appendix and secondary signs on ultrasound for pediatric appendicitis in the community hospital setting. Pediatr Surg Int 34:12871292

13. Reddan T, Corness J, Harden F, Mengersen K (2019) Improving the value of ultrasound in children with suspected appendicitis: a prospective study integrating secondary sonographic signs. Ultrasonography 38:67-75

14. Partain KN, Patel AU, Travers $C$ et al (2017) Improving ultrasound for appendicitis through standardized reporting of secondary signs. J Pediatr Surg 52:1273-1279
15. Unsdorfer KML, An JY, Binkovitz LA (2021) Pediatric appendiceal ultrasound: maintaining accuracy, increasing determinacy and improving clinical outcomes following the introduction of a standardized reporting template. Pediatr Radiol 51:265-272

16. Rodriguez DP, Vargas S, Callahan MJ et al (2006) Appendicitis in young children: imaging experience and clinical outcomes. AJR Am J Roentgenol 186:1158-1164

17. Samuel M (2002) Pediatric appendicitis score. J Pediatr Surg 37: 877-881

18. Rothrock SG, Pagane J (2000) Acute appendicitis in children: emergency department diagnosis and management. Ann Emerg Med 36:39-51

19. Lounis Y, Hugo J, Demarche M, Seghaye M-C (2020) Influence of age on clinical presentation, diagnosis delay and outcome in preschool children with acute appendicitis. BMC Pediatr 20:151

20. Dillman JR, Gadepalli S, Sroufe NS et al (2016) Equivocal pediatric appendicitis: unenhanced MR imaging protocol for nonsedated children - a clinical effectiveness study. Radiology 279:216-225

21. Reddan T, Corness J, Harden F, Mengersen K (2018) Paediatric appendiceal ultrasound: a survey of Australasian sonographers' opinions on examination performance and sonographic criteria. $\mathbf{J}$ Med Radiat Sci 65:267-274

22. Kessler N, Cyteval C, Gallix B et al (2004) Appendicitis: evaluation of sensitivity, specificity, and predictive values of US, Doppler US, and laboratory findings. Radiology 230:472-478

23. Trout AT, Sanchez R, Ladino-Torres MF (2012) Reevaluating the sonographic criteria for acute appendicitis in children. A review of the literature and a retrospective analysis of 246 cases. Acad Radiol 19:1382-1394

24. Lee MW, Kim YJ, Jeon HJ et al (2009) Sonography of acute right lower quadrant pain: importance of increased intraabdominal fat echo. AJR Am J Roentgenol 192:174-179

25. Serres SK, Cameron DB, Glass CC et al (2017) Time to appendectomy and risk of complicated appendicitis and adverse outcomes in children. JAMA Pediatr 171:740-746

26. Pham XD, Sullins VF, Kim DY et al (2016) Factors predictive of complicated appendicitis in children. J Surg Res 206:62-66

27. Singh M, Kadian YS, Rattan KN, Jangra B (2014) Complicated appendicitis: analysis of risk factors in children. Afr J Paediatr Surg 11:109-113

28. Cundy TP, Gent R, Frauenfelder C et al (2016) Benchmarking the value of ultrasound for acute appendicitis in children. J Pediatr Surg 51:1939-1943

Publisher's note Springer Nature remains neutral with regard to jurisdictional claims in published maps and institutional affiliations. 